\title{
New General Decay Rates of Solutions for Two Viscoelastic Wave Equations with Infinite Memory
}

\section{Aissa Guesmia}

Institut Elie Cartan de Lorraine, UMR 7502, Université de Lorraine

3 Rue Augustin Fresnel, BP 45112, 57073 Metz Cedex 03, France

E-mail(corresp.): aissa.guesmia@univ-lorraine.fr

Received June 10, 2019; revised March 14, 2020; accepted March 14, 2020

\begin{abstract}
We consider in this paper the problem of asymptotic behavior of solutions for two viscoelastic wave equations with infinite memory. We show that the stability of the system holds for a much larger class of kernels and get better decay rate than the ones known in the literature. More precisely, we consider infinite memory kernels $g: \mathbb{R}_{+}:=\left[0,+\infty\left[\rightarrow \mathbb{R}_{+}^{*}:=\right] 0,+\infty\left[\right.\right.$ satisfying $g^{\prime}(t) \leq-\xi(t) G(g(t)), \forall t \in \mathbb{R}_{+}$, where $\xi: \mathbb{R}_{+} \rightarrow \mathbb{R}_{+}^{*}$ and $G: \mathbb{R}_{+} \rightarrow \mathbb{R}_{+}$are given functions. Under this very general assumption on the behavior of $g$ at infinity and for each viscoelastic wave equation, we provide a relation between the decay rate of the solutions and the growth of $g$ at infinity, which improves the decay rates obtained in $[15,16,17,19,40]$. Moreover, we drop the boundedness assumptions on the history data considered in $[15,16,17,40]$.
\end{abstract}

Keywords: wave equation, infinite memory, asymptotic behavior, general decay.

AMS Subject Classification: 35B37; 35L55; 74D05; 93D15; 93D20.

\section{Introduction}

In this paper, we consider the following two viscoelastic problems:

$$
\begin{cases}u_{t t}(x, t)-\Delta u(x, t)+\int_{0}^{+\infty} g(s) \Delta u(x, t-s) d s=0 & \text { in } \Omega \times \mathbb{R}_{+}^{*}, \\ u(x, t)=0 & \text { on } \partial \Omega \times \mathbb{R}_{+}^{*}, \\ u(x,-t)=u_{0}(x, t), \quad u_{t}(x, 0)=u_{1}(x) & \text { in } \Omega \times \mathbb{R}_{+}\end{cases}
$$

Copyright (c) 2020 The Author(s). Published by VGTU Press

This is an Open Access article distributed under the terms of the Creative Commons Attribution License (http://creativecommons.org/licenses/by/4.0/), which permits unrestricted use, distribution, and reproduction in any medium, provided the original author and source are credited. 
and

$$
\begin{cases}u_{t t}(x, t)-\Delta u(x, t)-\int_{0}^{+\infty} g(s) u(x, t-s) d s=0 & \text { in } \Omega \times \mathbb{R}_{+}^{*}, \\ u(x, t)=0 & \text { on } \partial \Omega \times \mathbb{R}_{+}^{*}, \\ u(x,-t)=u_{0}(x, t), \quad u_{t}(x, 0)=u_{1}(x) & \text { in } \Omega \times \mathbb{R}_{+},\end{cases}
$$

where $u$ denotes the transverse displacement of waves, $\Delta$ is the Laplacian operator with respect to the space variable $x, u_{t}$ and $u_{t t}$ denote, respectively, the first and second derivatives with respect to the time variable $t, g: \mathbb{R}_{+} \rightarrow \mathbb{R}_{+}^{*}$ is a given function representing the infinite memory kernel and satisfying some hypotheses, $\Omega$ is a bounded domain of $\mathbb{R}^{N}, N \in \mathbb{N}^{*}:=\{1,2, \ldots\}$, with a smooth boundary $\partial \Omega$, and $u_{0}$ and $u_{1}$ are fixed history and initial data in a suitable Hilbert space.

\section{$1.1 \quad$ Finite memory}

The problems related to viscoelasticity (see [8]) have attracted a great deal of attention during the last four decades and many results of existence and longtime behavior have been established. Many advances in the studies of well posedness and stability were made since the works of Dafermos [10,11].

Hrusa [24] considered a one-dimensional nonlinear viscoelastic wave equation and proved several global existence results for large data and an exponential decay result for strong solutions when the kernel is of the form $g(s)=e^{-s}$. In [12], Dassios and Zafiropoulos considered a viscoelastic problem in $\mathbb{R}^{3}$ and proved a polynomial decay result for exponentially decaying kernels. After that, Rivera [38] considered equations for linear isotropic homogeneous viscoelastic solids of integral type which occupy bounded domains or the whole space $\mathbb{R}^{N}$. In the bounded-domain case and for exponentially decaying kernels and regular solutions, he showed that the sum of the first and the second energy decays exponentially. Whereas, the decay is polynomial when the body occupies the whole space $\mathbb{R}^{N}$, even if the kernel is of an exponential decay.

For quasilinear problems, Cavalcanti et al. [4] studied, in a bounded domain, the following equation:

$$
\left|u_{t}\right|^{\rho} u_{t t}-\Delta u-\Delta u_{t t}+\int_{0}^{t} g(t-s) \Delta u(s) d s-\gamma \Delta u_{t}=0 \quad \text { in } \Omega \times \mathbb{R}_{+}^{*},
$$

for $\rho>0$. A global existence result for $\gamma \geq 0$, as well as an exponential decay result for $\gamma>0$, have been established. This latter result was then extended to a situation, where $\gamma=0$, by Messaoudi and Tatar [31,32], and exponential and polynomial decay results have been established in the absence, as well as in the presence, of a source term. In all the above mentioned works, the rates of decay of kernels were either of exponential or polynomial type. In [6], Cavalcanti et al. considered a semilinear viscoelastic wave equation with local frictional damping, where the kernel $g$ satisfies, for two positive constants $\xi_{1}$ and $\xi_{2}$,

$$
-\xi_{1} g(t) \leq g^{\prime}(t) \leq-\xi_{2} g(t), \quad \forall t \in \mathbb{R}_{+}
$$


They established an exponential decay result under some restrictions on the control zone. Berrimi and Messaoudi [3] established the result of [6] under weaker conditions on both damping and kernel, for a problem where a source term is competing with the damping term. Cavalcanti and Oquendo [7] considered, in $\Omega \times \mathbb{R}_{+}^{*}$, the following problem:

$$
u_{t t}-k_{0} \Delta u+\int_{0}^{t} \operatorname{div}[a(x) g(t-s) \Delta u(s)] d s+b(x) h\left(u_{t}\right)+f(u)=0
$$

and established, for $a(x)+b(x) \geq \rho>0$, an exponential stability result for $g$ decaying exponentially and $h$ linear, and a polynomial stability result for $g$ decaying polynomially and $h$ nonlinear. Li et al. [27] treated (1.5) with $b(x)=0$ and $f(u)=-|u|^{\gamma} u, \gamma>0$. They showed the global existence and uniqueness of global solution of problem (1.5) and established uniform decay rate of the energy under suitable conditions on the initial data and $g$. For more general decaying kernels than the one defined by (1.4), Messaoudi [28,29] considered

$$
u_{t t}-\Delta u+\int_{0}^{t} g(t-s) \Delta u(s) d s=b|u|^{q-2} u \quad \text { in } \Omega \times \mathbb{R}_{+}^{*},
$$

for $q \geq 2, b \in\{0,1\}$ and $g$ satisfying, for a nonincreasing function $\xi: \mathbb{R}_{+} \rightarrow \mathbb{R}_{+}$,

$$
g^{\prime}(t) \leq-\xi(t) g(t), \quad \forall t \in \mathbb{R}_{+}
$$

He established a more general decay result, from which the usual exponential and polynomial decay rates are only special cases. Said-Houari et al. [39] studied the well-posedness and stability of coupled two semilinear viscoelastic wave equations, where the kernels satisfy (1.7). They established the same stability estimate as in $[28,29]$. Using the same assumption (1.7), a similar stability estimate was proved in [20] for a viscoelastic wave equation with Balakrishnan-Taylor damping and time-varying delay. Very recently, Messaoudi and Al-Khulaifi [30] considered (1.3) with $\gamma=0$, where the kernel satisfies, for $1 \leq p<\frac{3}{2}$,

$$
g^{\prime}(t) \leq-\xi(t) g^{p}(t), \quad \forall t \in \mathbb{R}_{+}
$$

and established a more general decay result, which leads to the optimal decay rate of solutions when $g$ converges to zero at ifinity faster than $s^{-2}$. Optimality means that the solution converges to zero as fast as $g$. Mustafa and Messaoudi [34] considered (1.6) with $b=0$ and kernels satisfying

$$
g^{\prime}(t) \leq-G(g(t)), \quad \forall t \in \mathbb{R}_{+}
$$

for some positive convex function $G: \mathbb{R}_{+} \rightarrow \mathbb{R}_{+}$. They used some properties of convex functions together with the generalized Young inequality and established a general decay result depending on $g$ and $G$. The assumption (1.9) was also considered in [22] for an abstract viscoelastic wave equation, where the obtained decay rate depends on the solution of an ODE. The results of [34] were generalized in [33] to kernels satisfying

$$
g^{\prime}(t) \leq-\xi(t) G(g(t)), \quad \forall t \in \mathbb{R}_{+}
$$


by getting a more general decay result depending on $g, \xi$ and $G$. This general decay result extendeds the range of the polynomial decay rate optimality from $p \in\left[1, \frac{3}{2}\right)$ to $p \in[1,2)$ in (1.8), which allows kernels having a decay at infinity of the form $g(s)=s^{-q}$, for $q>1$. The arguments of [33] were recently used in [23] to prove the stability of the following abstract equation:

$$
u_{t t}-A u-\int_{0}^{t} g(t-s) A^{\alpha} u(s) d s=0, \quad \forall t \in \mathbb{R}_{+}^{*},
$$

where $\alpha \in] 0,1[$ and $A$ is a given operator satisfying some hypotheses. For the case of memories acting on the boundary of domain, we refer the readers to $[5,14]$ and the references therein.

\subsection{Infinite memory}

Giorgi et al. [13] considered the following semilinear hyperbolic equation with infinite memory in a bounded domain $\Omega \subset \mathbb{R}^{3}$ :

$$
u_{t t}-K(0) \Delta u-\int_{0}^{+\infty} K^{\prime}(s) \Delta u(t-s) d s+g(u)=f \quad \text { in } \Omega \times \mathbb{R}_{+}^{*}
$$

under the condition $K^{\prime} \leq 0$ and proved the existence of global attractors for the solutions. Conti and Pata [9] added a linear frictional damping to (1.11) and assumed that the kernel is a convex decreasing smooth function. They proved the existence of a regular global attractor. Pata [35] discussed the decay properties of the semigroup generated by the following abstract equation:

$$
u_{t t}+\alpha A u(t)+\beta u_{t}(t)-\int_{0}^{+\infty} \mu(s) A u(t-s) d s=0, \quad \forall t \in \mathbb{R}_{+}^{*},
$$

where $A$ is a strictly positive self-adjoint linear operator, $\alpha>0, \beta \geq 0$ and $\mu$ is a decreasing function satisfying specific conditions. Subsequently, they established necessary as well as the sufficient conditions for the exponential stability. In [1], Appleby et al. studied (1.12) with $\alpha=1$ and $\beta=0$ and established an exponential decay result for strong solutions in a Hilbert space. In [15], Guesmia considered the abstract equation

$$
u_{t t}+A u-\int_{0}^{+\infty} g(s) B u(t-s) d s=0, \quad \forall t \in \mathbb{R}_{+}^{*}
$$

in a Hilbert space $H$, where $A$ and $B$ are positive self-adjoint linear operators such that $A \sim B$ or $A$ is stronger than $B$ (in some sense). Under the boundedness conditions on the first initial data

$$
\begin{cases}\sup _{s \in \mathbb{R}_{+}}\left\|B^{\frac{1}{2}} u_{0}(s)\right\|_{H}<+\infty, & \text { if } A \sim B, \\ \sup _{s \in \mathbb{R}_{+}}\left\|A^{\frac{1}{2}} B^{\frac{1}{2}} u_{0}(s)\right\|_{H}<+\infty, & \text { if } A \text { is stronger than } B\end{cases}
$$

and for the larger class of kernels satisfying

$$
\int_{0}^{+\infty} \frac{g(s)}{G^{-1}\left(-g^{\prime}(s)\right)} d s+\sup _{s \in \mathbb{R}^{+}} \frac{g(s)}{G^{-1}\left(-g^{\prime}(s)\right)}<+\infty,
$$


where $G: \mathbb{R}_{+} \rightarrow \mathbb{R}_{+}$is an increasing strictly convex function, he proved two general decay results corresponding to the cases $A \sim B$ and $A$ is stronger than $B$. Using this approach, Guesmia and Messaoudi [18] later looked into

$u_{t t}-\Delta u+\int_{0}^{t} g_{1}(t-s) \operatorname{div}\left(a_{1}(x) \nabla u(s)\right) d s+\int_{0}^{+\infty} g_{2}(s) \operatorname{div}\left(a_{2}(x) \nabla u(t-s)\right) d s=0$

under the first condition in (1.14) (with $B=-\Delta$ ) and suitable conditions on $a_{1}$ and $a_{2}$ and for a wide class of kernels $g_{1}$ and $g_{2}$ satisfying (1.7) and (1.15), respectively. They established a general decay result including, in particular, the usual cases of exponential and polynomial decay. The decay rate of solutions obtained in [15] and [18] is weaker than the one of $g$ at infinity when $g$ does not converge exponentially to zero. The authors of [17] considered (1.13) with $A=B$ and under (1.7) and the first condition in (1.14). They proved a general decay result of the solution depending only on $g$ and $\xi$ by adopting, for infinite memory, the method introduced in [28] with some modifications imposed by the nature of their problem. In the particular cases where $g$ converges to zero at infinity faster than any polynomial, the obtained decay rate of solutions in [17] is equal to the one of $g$; and so it improves the one presented in [15]. A similar stability result was proved in [21] for an abstract thermoelastic system by considering (1.7) and applying the arguments of [17]. Recently, Youkana [40] considered (1.13) in which $g$ and $u_{0}$ satisfy (1.8) and (1.14). He obtained a better decay rate than the one of [15] and [17] when $g$ has a decay at infinity of the form $s^{-q}$, for $q>2$.

In [16], the problem of indirect stability of two coupled abstract equations with one infinite memory was considered. More precisely, the author of [16] studied

$$
\begin{cases}u_{t t}(t)+A u(t)-\int_{0}^{+\infty} g(s) B u(t-s) d s+\tilde{B} v(t)=0, & \forall t \in \mathbb{R}_{+}^{*}, \\ v_{t t}(t)+\tilde{A} v(t)+\tilde{B} u(t)=0, & \forall t \in \mathbb{R}_{+}^{*}\end{cases}
$$

in a Hilbert space $H$, where $A, \tilde{A}, B$ and $\tilde{B}$ are positive self-adjoint linear operators such that $A \sim B$ or $A$ is stronger than $B$, and $g$ satisfies (1.15). Under the following weaker condition on $u_{0}$ than (1.14):

$$
\sup _{t \in \mathbb{R}_{+}} \int_{t}^{+\infty} \frac{g(s)}{G^{-1}\left(-g^{\prime}(s)\right)}\left\|A^{\frac{j}{2}} B^{\frac{1}{2}} u_{0}(s-t)\right\|_{H}^{2} d s<+\infty
$$

where $j=0$ if $A \sim B$, and $j=1$ if $A$ is stronger than $B$, it was proved a general decay estimte depending also on the smoothness of initial data. For classical solutions, the estimate of [16] coincides with the one of [15] when $A$ is stronger than $B$. The stability of the same system (1.16) was the subject of the paper [26], where the authors proved that the decay rate $\frac{1}{t}$ of the energy is guaranteed by the following weak condition on the decreasingness behavior of $g$ :

$$
\mid\left\{s \in \mathbb{R}_{+}: g(s)>0 \text { and } g^{\prime}(s)=0\right\} \mid=0,
$$

(here $|\cdot|$ means the Lebesgue measure). The same result of [26] was proved in [25] for (1.16) with a finite memory (instead of the infinite one) and an additional semilinear term $f(u)$ on the first equation. More precisely, the authors 
of [25] considered (1.18) and obtained the decay rate $\frac{1}{t}$ of energy for the coupled system as well as for the corresponding first single equation.

Without assuming any boundedness condition on $u_{0}$ and for $g$ satisfying

$$
g(t-s) \geq \xi(t) \int_{t}^{+\infty} g(\tau-s) d \tau, \quad \forall t \in \mathbb{R}_{+}, \forall s \in[0, t],
$$

the authors of [19] proved the well posedness and stability of (1.13) with a distributed delay; that is

$$
u_{t t}+A u-\int_{0}^{+\infty} g(s) B u(t-s) d s+\int_{0}^{+\infty} f(s) u_{t}(t-s) d s=0, \quad t \in \mathbb{R}_{+}^{*},
$$

where $A \sim B$ and $f: \mathbb{R}_{+} \rightarrow \mathbb{R}$ is dominated by $g$ (in some sense). The stability results of [19] lead to the same decay rate of [17] when $g$ converges to zero at infinity faster than $t^{-2}$. However, when the decay rate of $g$ is at most of the form $t^{-2}$, no stability result is obtained in [19].

In the present work, we study the asymptotic behavior of solutions of (1.1) and (1.2) as particular models of (1.13) corresponding to the cases, respectively, $A \sim B$ and $A$ is stronger than $B$. Under the general assumption (1.10) instead of $(1.15),(1.7),(1.19)$ and $(1.8)$ considered in $[15,17,19,40]$, respectively, we prove two general decay estimates of solutions, which improve the decay rates obtained in $[15,16,17,19,40]$ in case when $g$ has at most a polynomial decay at infinity (see examples in Section 4 ). Moreover, our class of admissible initial data is larger than the one considered in $[15,16,17,40]$ because we do not assume any boundedness condition on $u_{0}$.

The rest of this paper is organized as follows. In Section 2, we present some assumptions and material needed for our work and give the well posedness results of our two systems. Some technical lemmas are presented and proved in Section 3. Finally, we state and prove our main decay results and provide some examples in Section 4.

\section{Assumptions and well posedness}

In this section, we present some materials needed for the proof of our results and state the well-posedness results of (1.1) and (1.2). We use the standard Lebesgue space $L^{2}(\Omega)$ and Sobolev space $H_{0}^{1}(\Omega)$ with their usual scalar products that generate the norms $\|\cdot\|_{2}$ and $\|\nabla \cdot\|_{2}$, respectively. We assume the following hypotheses:

(A1) $g: \mathbb{R}_{+} \rightarrow \mathbb{R}_{+}^{*}$ is a $C^{1}\left(\mathbb{R}_{+}\right)$nonincreasing function satisfying, for some $\beta_{0}>0$,

$$
-\beta_{0} g(s) \leq g^{\prime}(s), \forall s \in \mathbb{R}_{+} \quad \text { and } \begin{cases}1-g_{0}:=\ell>0 & \text { in case (1.1), } \\ 1-c_{0} g_{0}:=\ell>0 & \text { in case (1.2), }\end{cases}
$$

where $g_{0}:=\int_{0}^{+\infty} g(s) d s$ and $c_{0}$ is the smallest constant satisfying (Poincaré's inequality)

$$
\|v\|_{2}^{2} \leq c_{0}\|\nabla v\|_{2}^{2}, \quad \forall v \in H_{0}^{1}(\Omega)
$$


(A2) There exists a function $G: \mathbb{R}_{+} \rightarrow \mathbb{R}_{+}$in $C^{1}\left(\mathbb{R}_{+}\right) \cap C^{2}\left(\mathbb{R}_{+}^{*}\right)$ which is increasing and strictly convex, with

$$
G(0)=G^{\prime}(0)=0, \quad \lim _{s \rightarrow+\infty} G^{\prime}(s)=+\infty,
$$

$s \mapsto s G^{\prime}(s)$ and $s \mapsto s\left(G^{\prime}\right)^{-1}(s)$ are convex on $\mathbb{R}_{+}$, and there exists a nonincreasing function $\xi: \mathbb{R}_{+} \rightarrow \mathbb{R}_{+}^{*}$ in $C^{1}\left(\mathbb{R}_{+}\right)$such that

$$
\int_{0}^{+\infty} \xi(s) d s=+\infty, \quad g^{\prime}(s) \leq-\xi(s) G(g(s)), \quad \forall s \in \mathbb{R}_{+} .
$$

Remark 1. As a simple example of functions $g$ satisfying (A1) and (A2), we can take

$$
g(s)=a(1+s)^{-q},
$$

for $q>1,0<a<q-1$ in case (1.1), and $0<a<\frac{q-1}{c_{0}}$ in case (1.2), with $\beta_{0}=q, \xi(s)=q a^{-\frac{1}{q}}$ and $G(s)=s^{\frac{q+1}{q}}$.

Now, we state the existence results to (1.1) and (1.2); the proof is given in [15]. Systems (1.1) and (1.2) can be formulated as the following abstract linear first order system:

$$
\left\{\begin{array}{l}
\mathcal{U}^{\prime}(t)=\mathcal{A} \mathcal{U}(t), \quad \forall t \in \mathbb{R}_{+}^{*} \\
\mathcal{U}(0)=\mathcal{U}_{0}
\end{array}\right.
$$

where $\mathcal{U}_{0}=\left(u_{0}(\cdot, 0), u_{1}, \eta_{0}(\cdot, s)\right)^{T} \in \mathcal{H}, \mathcal{H}=H_{0}^{1}(\Omega) \times L^{2}(\Omega) \times L_{g}^{i}, \mathcal{U}=$ $\left(u, u^{\prime}, \eta\right)^{T}, i=1$ for $(1.1), i=0$ for $(1.2)$,

$$
\begin{cases}\eta(x, t, s)=u(x, t)-u(x, t-s), & \forall(x, t, s) \in \Omega \times \mathbb{R}_{+} \times \mathbb{R}_{+}, \\ \eta_{0}(x, s)=u_{0}(x, 0)-u_{0}(x, s), & \forall(x, s) \in \Omega \times \mathbb{R}_{+},\end{cases}
$$

( $\eta^{t}$ is the relative history of $u$, and it was introduced first in [11]), $L_{g}^{i}$ is the weighted space with respect to the measure $g(s) d s$ defined by

$$
L_{g}^{i}=\left\{z: \mathbb{R}_{+} \rightarrow H_{0}^{i}(\Omega), \int_{0}^{+\infty} g(s)\left\|\nabla^{i} z(s)\right\|^{2} d s<+\infty\right\}
$$

where we note $H_{0}^{0}(\Omega)=L^{2}(\Omega), \nabla^{0}=I d, \nabla^{1}=\nabla, I d$ is the identity operator and $\mathcal{A}$ is the linear operator given by

$$
\mathcal{A}(v, w, z)^{T}=\left(w,\left(1-g_{0}\right) \Delta v+\int_{0}^{+\infty} g(s) \Delta z(s) d s,-z_{s}+w\right)^{T}
$$

in case $(1.1)$, where $z_{s}=\frac{\partial z}{\partial s}$,

$$
\mathcal{A}(v, w, z)^{T}=\left(w, \Delta v+g_{0} v-\int_{0}^{+\infty} g(s) z(s) d s,-z_{s}+w\right)^{T}
$$


in case (1.2), and

$$
D(\mathcal{A})=\left\{(v, w, z)^{T} \in \mathcal{H}, \mathcal{A}(v, w, z)^{T} \in \mathcal{H}, z(0)=0\right\} .
$$

Under the assumption (A1), the space $\mathcal{H}$ is a Hilbert space, $D(\mathcal{A}) \subset \mathcal{H}$ with dense embedding, and $\mathcal{A}$ is the infinitesimal generator of a linear contraction $C_{0}$-semigroup on $\mathcal{H}$ (see [15]). Therefore, from the classical semigroup theory (see [36]), we get the following well-posedness results for (2.3) (see [15] with $(A, B)=(-\Delta,-\Delta)$ for $(1.1)$, and $(A, B)=(-\Delta, I d)$ for $(1.2))$ :

Theorem 1. [15] Assume that (A1) is satisfied. Then, for any $\mathcal{U}_{0} \in \mathcal{H},(2.3)$ has a unique weak solution $\mathcal{U} \in C\left(\mathbb{R}_{+}, \mathcal{H}\right)$. Moreover, if $\mathcal{U}_{0} \in D(\mathcal{A})$, then the solution of $(2.3)$ is classical; that is $\mathcal{U} \in C^{1}\left(\mathbb{R}_{+}, \mathcal{H}\right) \cap C\left(\mathbb{R}_{+}, D(\mathcal{A})\right)$.

Consequently, by assuming that (A1) is satisfied, the above theorem implies that, for any $\left(u_{0}(\cdot, 0), u_{1}\right) \in H_{0}^{1}(\Omega) \times L^{2}(\Omega)$ such that $\eta_{0} \in L_{g}^{i},(1.1)$ and $(1.2)$ have a unique global (weak) solution $u \in C\left(\mathbb{R}_{+}, H_{0}^{1}(\Omega)\right) \cap C^{1}\left(\mathbb{R}_{+}, L^{2}(\Omega)\right)$. Moreover, if $\left(u_{0}(\cdot, 0), u_{1}\right) \in\left(H^{2}(\Omega) \cap H_{0}^{1}(\Omega)\right) \times H_{0}^{1}(\Omega)$ such that $\eta_{0}, \frac{\partial \eta_{0}}{\partial s} \in L_{g}^{i}$, then the solution $u$ satisfies (classical solution)

$$
u \in C\left(\mathbb{R}_{+}, H^{2}(\Omega) \cap H_{0}^{1}(\Omega)\right) \cap C^{1}\left(\mathbb{R}_{+}, H_{0}^{1}(\Omega)\right) \cap C^{2}\left(\mathbb{R}_{+}, L^{2}(\Omega)\right) .
$$

Now, we consider the assumptions (A1) and (A2) and take initial data $\left(u_{0}, u_{1}\right)$ such that

$$
\left(u_{0}(\cdot, 0), u_{1}, \eta_{0}\right) \in H_{0}^{1}(\Omega) \times L^{2}(\Omega) \times L_{g}^{1}
$$

for (1.1), and

$$
\left\{\begin{array}{l}
\left(u_{0}(\cdot, 0), u_{1}, \eta_{0}\right) \in H_{0}^{1}(\Omega) \times L^{2}(\Omega) \times L_{g}^{0}, \\
\left(\nabla u_{0}(\cdot, 0), \nabla u_{1}, \nabla \eta_{0}\right) \in\left(H_{0}^{1}(\Omega)\right)^{N} \times\left(L^{2}(\Omega)\right)^{N} \times\left(L_{g}^{0}\right)^{N}
\end{array}\right.
$$

for (1.2). We introduce the "modified" energy associated to (1.1) and (1.2)

$$
E(t)=\frac{1}{2}\left\|u_{t}\right\|_{2}^{2}+\frac{1}{2}\|\nabla u\|_{2}^{2}-\frac{g_{0}}{2}\|f\|_{2}^{2}+\frac{1}{2}(g \circ f)(t),
$$

where $f=\nabla u$ for (1.1), $f=u$ for (1.2) and we use the notation

$$
(g \circ f)(t)=\int_{0}^{+\infty} g(s)\|f(t)-f(t-s)\|_{2}^{2} d s .
$$

Also, following the idea of [37], we introduce the second "modified" energy associated to (1.2)

$$
E_{*}(t)=\frac{1}{2}\left\|\nabla u_{t}\right\|_{2}^{2}+\frac{1}{2}\|\Delta u\|_{2}^{2}-\frac{g_{0}}{2}\|\nabla u\|_{2}^{2}+\frac{1}{2}(g o \nabla u)(t) .
$$

Direct differentiation (multiplying $(1.1)_{1}$ by $u_{t}$, multiplying $(1.2)_{1}$, first, by $u_{t}$ and, second, by $\Delta u_{t}$, integrating by parts and using boundary conditions; see [37]) leads to

$$
E^{\prime}(t)=\frac{1}{2}\left(g^{\prime} \circ f\right)(t) \leq 0
$$


and

$$
E_{*}^{\prime}(t)=\frac{1}{2}\left(g^{\prime} o \nabla u\right)(t) \leq 0 .
$$

On the other hand, using (2.1) and (2.2), we get

$$
\frac{\ell}{2}\left(\left\|u_{t}\right\|_{2}^{2}+\|\nabla u\|_{2}^{2}+(g \circ f)(t)\right) \leq E(t) \leq \frac{1}{2}\left(\left\|u_{t}\right\|_{2}^{2}+\|\nabla u\|_{2}^{2}+(g \circ f)(t)\right)
$$

and

$$
\frac{\ell}{2}\left(\left\|\nabla u_{t}\right\|_{2}^{2}+\|\Delta u\|_{2}^{2}+(g o \nabla u)(t)\right) \leq E_{*}(t) \leq \frac{1}{2}\left(\left\|\nabla u_{t}\right\|_{2}^{2}+\|\Delta u\|_{2}^{2}+(g o \nabla u)(t)\right) .
$$

\section{Preliminaries lemmas}

In this section, we assume that (A1), (A2), (2.4) and (2.5) are satisfied, and we establish several lemmas needed for the proof of our stability results, which will be presented and proved in Section 4. These lemmas allow us to deal with the finite and infinite parts of the infinite memory. For the finite part, we apply the approach developped in [30] and [33] for the stability of wave equations with finite memory.

Lemma 1. There exists a positive constant $M_{0}$ such that, for any $t \in \mathbb{R}_{+}$,

$$
\int_{t}^{+\infty} g(s)\|\nabla u(t)-\nabla u(t-s)\|_{2}^{2} d s \leq M_{0} \int_{0}^{+\infty} g(t+s)\left(1+\left\|\nabla u_{0}(s)\right\|_{2}^{2}\right) d s .
$$

Proof. Using (2.6) and the left inequality in (2.8), we obtain, for any $t \in \mathbb{R}_{+}$,

$$
\begin{aligned}
& \int_{t}^{+\infty} g(s)\|\nabla u(t)-\nabla u(t-s)\|_{2}^{2} d s \\
& \quad \leq 2\|\nabla u(t)\|_{2}^{2} \int_{t}^{+\infty} g(s) d s+2 \int_{t}^{+\infty} g(s)\|\nabla u(t-s)\|_{2}^{2} d s \\
& \quad \leq 2 \sup _{s \geq 0}\|\nabla u(s)\|_{2}^{2} \int_{0}^{+\infty} g(t+s) d s+2 \int_{0}^{+\infty} g(t+s)\|\nabla u(-s)\|_{2}^{2} d s \\
& \quad \leq \frac{4}{\ell} \sup _{s \geq 0} E(s) \int_{0}^{+\infty} g(t+s) d s+2 \int_{0}^{+\infty} g(t+s)\left\|\nabla u_{0}(s)\right\|_{2}^{2} d s \\
& \quad \leq \frac{4}{\ell} E(0) \int_{0}^{+\infty} g(t+s) d s+2 \int_{0}^{+\infty} g(t+s)\left\|\nabla u_{0}(s)\right\|_{2}^{2} d s,
\end{aligned}
$$

which gives (3.1) with $M_{0}=\max \left\{\frac{4}{\ell} E(0), 2\right\}$.

\section{Lemma 2. Let}

$$
\psi_{1}(t)=\int_{\Omega} u u_{t} d x \quad \text { and } \quad \psi_{2}(t)=-\int_{\Omega} u_{t} \int_{0}^{+\infty} g(s)(u(t)-u(t-s)) d s d x .
$$

Then there exist positive constants $N, N_{1}, N_{2}, a_{1}, a_{2}$ such that the functional

$$
L=N\left(E+\left(1-\delta_{0}\right) E_{*}\right)+N_{1} \psi_{1}+N_{2} \psi_{2},
$$


where $\delta_{0}=1$ for (1.1), and $\delta_{0}=0$ for (1.2), satisfies

$$
\begin{aligned}
& a_{1} E \leq L \leq a_{2} E \quad \text { for }(1.1), \quad L \geq a_{1} E \quad \text { for (1.2) } \\
& L^{\prime}(t) \leq-4(1-\ell)\|\nabla u\|_{2}^{2}-\left\|u_{t}\right\|_{2}^{2}+\frac{\tau_{0}}{4}(g \circ \nabla u)(t), \quad \forall t \in \mathbb{R}_{+},
\end{aligned}
$$

where $\tau_{0}=1$ for (1.1), and $\tau_{0}=c_{0}$ for (1.2).

Proof. This lemma can be obtained by a direct application of the arguments of [33] used for (1.1) with finite memory. For (1.2), we need only to use Poincaré's inequality $(2.2)$ to estimate $(g o u)(t)$ by $c_{0}(g o \nabla u)(t)$.

Lemma 3. Let

$$
\psi_{3}(t)=\int_{0}^{t} \psi_{4}(t-s)\|\nabla u(s)\|_{2}^{2} d s \quad \text { and } \quad \psi_{4}(t)=\int_{t}^{+\infty} g(s) d s .
$$

Then, for any $t \in \mathbb{R}_{+}$,

$$
\psi_{3}^{\prime}(t) \leq-\frac{1}{2} \int_{0}^{t} g(s)\|\nabla u(t)-\nabla u(t-s)\|_{2}^{2} d s+\frac{3}{\tau_{0}}(1-\ell)\|\nabla u(t)\|_{2}^{2} .
$$

Proof. The proof of this lemma is also a direct application of the arguments of [33]. Indeed, we have $\psi_{4}^{\prime}=-g, \psi_{4}(0)=g_{0}$ and

$$
\int_{0}^{t} g(t-s) d s=\int_{0}^{t} g(s) d s=g_{0}-\psi_{4}(t)
$$

( $g_{0}$ is defined in hypothesis (A1)), then

$$
\begin{aligned}
& \psi_{3}^{\prime}(t)=\psi_{4}(0)\|\nabla u(t)\|_{2}^{2}+\int_{0}^{t} \psi_{4}^{\prime}(t-s)\|\nabla u(s)\|_{2}^{2} d s=g_{0}\|\nabla u(t)\|_{2}^{2} \\
& -\int_{0}^{t} g(t-s)\|\nabla u(s)\|_{2}^{2} d s=\psi_{4}(t)\|\nabla u(t)\|_{2}^{2}-\int_{0}^{t} g(t-s) \| \nabla u(t) \\
& -\nabla u(s) \|_{2}^{2} d s+2 \int_{0}^{t} g(t-s)\langle\nabla u(t), \nabla u(t)-\nabla u(s)\rangle d s
\end{aligned}
$$

where $\langle$,$\rangle denotes the standard scalar product in \left(L^{2}(\Omega)\right)^{n}$. Using Young's and Hölder's inequalities for the last term of the above equality, we get

$$
\begin{gathered}
2 \int_{0}^{t} g(t-s)\langle\nabla u(t), \nabla u(t)-\nabla u(s)\rangle d s \leq \frac{2(1-\ell)}{\tau_{0}}\|\nabla u(t)\|_{2}^{2} \\
+\frac{\tau_{0} \int_{0}^{t} g(s) d s}{2(1-\ell)} \int_{0}^{t} g(t-s)\|\nabla u(t)-\nabla u(s)\|_{2}^{2} d s
\end{gathered}
$$

where $\ell$ and $\tau_{0}$ are defined in (2.1) and Lemma 2, respectively. Because

$$
\psi_{4}(t) \leq \psi_{4}(0)=g_{0}=\frac{1-\ell}{\tau_{0}} \quad \text { and } \quad \frac{\tau_{0} \int_{0}^{t} g(s) d s}{2(1-\ell)} \leq \frac{\tau_{0} g_{0}}{2(1-\ell)}=\frac{1}{2},
$$

then by combining the above three inequalities, we get (3.4). 
Lemma 4. There exits a positive constant $\tilde{b}$ such that the energy functional satisfies

$$
\int_{0}^{t} E(s) d s<\tilde{b}(\tilde{h}(t)+1), \quad \forall t \in \mathbb{R}_{+}
$$

where

$$
\tilde{h}(t)=\int_{0}^{t} \int_{0}^{+\infty} g(s+\tau)\left(1+\left\|\nabla u_{0}(\tau)\right\|_{2}^{2}\right) d \tau d s, \quad t \in \mathbb{R}_{+} .
$$

Proof. Let $F=L+\tau_{0} \psi_{3}$, then using (3.3) and (3.4), we obtain

$$
\begin{aligned}
F^{\prime}(t) \leq & -(1-\ell)\|\nabla u\|_{2}^{2}-\left\|u_{t}\right\|_{2}^{2}-\frac{\tau_{0}}{4}(g o \nabla u)(t) \\
& +\frac{\tau_{0}}{2} \int_{t}^{+\infty} g(s)\|\nabla u(t)-\nabla u(t-s)\|_{2}^{2} d s, \quad \forall t \in \mathbb{R}_{+} .
\end{aligned}
$$

Using (2.2) (for $(g o u)(t))$ in case (1.2), the right inequality in (2.8) and (3.7), we see that there exists a positive constant $b=2 \min \left\{1-l, 1, \frac{1}{4}\right\}$ satisfying

$$
F^{\prime}(t) \leq-b E(t)+\frac{\tau_{0}}{2} \int_{t}^{+\infty} g(s)\|\nabla u(t)-\nabla u(t-s)\|_{2}^{2} d s, \quad \forall t \in \mathbb{R}_{+} .
$$

Therefore, using (3.1) and noting that $F \geq 0$ (since $\psi_{3} \geq 0$ and $L \geq 0$ thanks to $(3.2))$,

$$
b \int_{0}^{t} E(s) d s \leq F(0)-F(t)+\frac{\tau_{0} M_{0}}{2} \tilde{h}(t) \leq F(0)+\frac{\tau_{0} M_{0}}{2} \tilde{h}(t), \quad \forall t \in \mathbb{R}_{+} .
$$

Hence (3.5) is proved with $\tilde{b}=\frac{1}{b} \max \left\{F(0), \frac{\tau_{0} M_{0}}{2}\right\}$.

Lemma 5. There exists $0<\epsilon_{0}<1$ such that, for any $t \in \mathbb{R}_{+}$,

$$
\int_{0}^{t} g(s)\|\nabla u(t)-\nabla u(t-s)\|_{2}^{2} d s \leq \frac{\tilde{h}(t)+1}{\epsilon_{0}} G^{-1}\left(\frac{\epsilon_{0} I(t)}{\xi(t)(\tilde{h}(t)+1)}\right),
$$

where

$$
I(t)=-\int_{0}^{t} g^{\prime}(s)\|\nabla u(t)-\nabla u(t-s)\|_{2}^{2} d s .
$$

Proof. Using (2.6), the left inequality in (2.8) and (3.5), we obtain

$$
\begin{aligned}
& \int_{0}^{t}\|\nabla u(t)-\nabla u(t-s)\|_{2}^{2} d s \leq 2 \int_{0}^{t}\left(\|\nabla u(t)\|_{2}^{2}+\|\nabla u(t-s)\|_{2}^{2}\right) d s \\
& \quad \leq \frac{4}{\ell} \int_{0}^{t}(E(t)+E(t-s)) d s \leq \frac{8}{\ell} \int_{0}^{t} E(s) d s \leq \frac{8 \tilde{b}}{\ell}(\tilde{h}(t)+1), \quad \forall t \in \mathbb{R}_{+} .
\end{aligned}
$$

Then, for $0<\epsilon_{0}<\min \{1, \ell / 8 \tilde{b}\}$, we get $q_{0}(t):=\frac{\epsilon_{0}}{\tilde{h}(t)+1}<1 \quad$ and $\quad q_{0}(t) \int_{0}^{t}\|\nabla u(t)-\nabla u(t-s)\|_{2}^{2} d s<1, \quad \forall t \in \mathbb{R}_{+}$.

So, the proof of (3.8) is the one given in [33]. 
Remark 2. Using (2.6) and (2.7), we notice that

$$
I \leq-2\left(\delta_{0} E^{\prime}+\left(1-\delta_{0}\right) E_{*}^{\prime}\right),
$$

where $\delta_{0}=1$ for (1.1), and $\delta_{0}=0$ for (1.2).

\section{Stability results}

Before presenting our stability results, we put

$$
\begin{aligned}
& G_{0}(t)=t G^{\prime}(t), \quad G_{1}(t)=\int_{t}^{1} \frac{1}{G_{0}(s)} d s, \quad G_{2}(t)=t\left(G^{\prime}\right)^{-1}(t), \\
& h(t)=\int_{0}^{+\infty} g(t+s)\left(1+\left\|\nabla u_{0}(s)\right\|_{2}^{2}\right) d s .
\end{aligned}
$$

On the other hand, for fixed positive constants $c_{1}$ and $c_{2}$, we introduce the class of functions $\alpha: \mathbb{R}_{+} \rightarrow \mathbb{R}_{+}^{*}$ satisfying

$$
\begin{aligned}
& \alpha \in C^{1}\left(\mathbb{R}_{+}\right), \quad \alpha \leq 1, \quad \alpha^{\prime} \leq 0 \\
& c_{2} G_{2}^{*}\left(\frac{c_{2} h(t)}{\tilde{h}(t)+1}\right) \leq c_{1}\left(G_{0}\left(\frac{\gamma(t)}{\alpha(t)}\right)-\frac{G_{0}(\gamma(t))}{\alpha(t)}\right), \quad \forall t \in \mathbb{R}_{+},
\end{aligned}
$$

where

$$
\gamma(t)=G_{1}^{-1}\left(c_{1} \int_{0}^{t} \xi(s) d s\right), \quad t \in \mathbb{R}_{+},
$$

$\tilde{h}$ is defined in (3.6); that is $\tilde{h}(t)=\int_{0}^{t} h(s) d s$, and $G_{2}^{*}(t)=\sup _{s \in \mathbb{R}_{+}}\left\{t s-G_{2}(s)\right\}$, $t \in \mathbb{R}_{+}$is the convex conjugate of $G_{2}$ in the sense of Young (see [2]). Thanks to (A2), $G_{2}^{*}$ is given by

$$
G_{2}^{*}(t)=t\left(G_{2}^{\prime}\right)^{-1}(t)-G_{2}\left[\left(G_{2}^{\prime}\right)^{-1}(t)\right], \quad t \in \mathbb{R}_{+} .
$$

According to (A2), $G_{0}$ is convex increasing on $\mathbb{R}_{+}$and defines a bijection from $\mathbb{R}_{+}$to $\mathbb{R}_{+}, G_{1}$ is decreasing and defines a bijection from $\left.] 0,1\right]$ to $\mathbb{R}_{+}, G_{2}$ is convex and increasing on $\mathbb{R}_{+}, \gamma$ is of class $C^{1}$ on $\mathbb{R}_{+}$, and $G_{2}^{*}$ is convex and increasing on $\mathbb{R}_{+}$. Moreover, $h$ is a $C^{1}\left(\mathbb{R}_{+}\right)$because, in both cases (1.1) and (1.2), we have $\eta_{0} \in L_{g}^{1}$; so, for any $t \in \mathbb{R}_{+}$,

$$
\begin{gathered}
\int_{0}^{+\infty} g(t+s)\left(1+\left\|\nabla u_{0}(s)\right\|_{2}^{2}\right) d s \leq g_{0}+\int_{0}^{+\infty} g(t+s)\left\|\nabla u_{0}(0)-\nabla \eta_{0}(s)\right\|_{2}^{2} d s \\
\leq g_{0}\left(1+2\left\|\nabla u_{0}(0)\right\|_{2}^{2}\right)+2 \int_{0}^{+\infty} g(s)\left\|\nabla \eta_{0}(s)\right\|_{2}^{2} d s:=M_{1}, \quad \forall t \in \mathbb{R}_{+}
\end{gathered}
$$

(since $\eta_{0} \in L_{g}^{1}$ thanks to (2.4) and (2.5)) and, using the left assumption in $(2.1)$,

$$
\begin{aligned}
\left|h^{\prime}(t)\right| & =-\int_{0}^{+\infty} g^{\prime}(t+s)\left(1+\left\|\nabla u_{0}(s)\right\|_{2}^{2}\right) d s \\
& \leq \beta_{0} \int_{0}^{+\infty} g(t+s)\left(1+\left\|\nabla u_{0}(s)\right\|_{2}^{2}\right) d s=\beta_{0} h(t) \leq \beta_{0} M_{1}, \quad \forall t \in \mathbb{R}_{+} .
\end{aligned}
$$

Finally, we remark that $\tilde{h}$ is of class $C^{1}$ and increasing on $\mathbb{R}_{+}$. 
Theorem 2. Assume that (A1), (A2), (2.4) and (2.5) are satisfied. Then there exist positive constants $c_{1}, c_{2}$ and $c_{3}$ such that, for any $\alpha$ satisfying (4.3) and (4.4), the solution of (1.1) satisfies

$$
E(t) \leq c_{3}(\tilde{h}(t)+1) \gamma(t) / \alpha(t), \quad \forall t \in \mathbb{R}_{+},
$$

and the solution of (1.2) satisfies, for any $t \in \mathbb{R}_{+}^{*}$,

$$
E(t) \leq c_{1}(\tilde{h}(t)+1) G_{0}^{-1}\left(\frac{c_{1}\left(1+\int_{0}^{t} \xi(s) G_{2}^{*}\left(\frac{c_{1} h(s)}{\tilde{h}(s)+1}\right) d s\right)}{\int_{0}^{t} \xi(s) d s}\right)
$$

Proof. To simplify the computations, we note by $c$ and $\hat{c}$ two generic positive constants which may change from line to line. Without lose of generality, we assume that $E(0)>0$ (otherwise, $E \equiv 0$ because $E$ is nonnegative and nonincreasing). For the proof of (4.7) and (4.8), combining (3.3) and the right inequality in (2.8), and using (2.2) for $(g o u)(t)$ in case (1.2), we obtain

$$
\begin{aligned}
L^{\prime}(t) \leq-c E(t) & +\hat{c} \int_{0}^{t} g(s)\|\nabla u(t)-\nabla u(t-s)\|_{2}^{2} d s \\
& +\hat{c} \int_{t}^{+\infty} g(s)\|\nabla u(t)-\nabla u(t-s)\|_{2}^{2} d s, \quad \forall t \in \mathbb{R}_{+} .
\end{aligned}
$$

Recalling (3.1) and the definition of $h$ in (4.2), we get

$$
L^{\prime}(t) \leq-c E(t)+\hat{c} \int_{0}^{t} g(s)\|\nabla u(t)-\nabla u(t-s)\|_{2}^{2} d s+\hat{c} h(t), \quad \forall t \in \mathbb{R}_{+} .
$$

Combining (4.10) and (3.8), we get

$$
L^{\prime}(t) \leq-c E(t)+\frac{\hat{c}(\tilde{h}(t)+1)}{\epsilon_{0}} G^{-1}\left(\frac{\epsilon_{0} I(t)}{\xi(t)(\tilde{h}(t)+1)}\right)+\hat{c} h(t), \quad \forall t \in \mathbb{R}_{+} .
$$

The functional $\mathcal{F}$ defined, for fixed $\epsilon>0$, by

$$
\mathcal{F}=G^{\prime}(\varepsilon E /(E(0)(\tilde{h}+1))) L
$$

satisfies, for any $t \in \mathbb{R}_{+}$(noting that $G^{\prime \prime} \geq 0, \tilde{h}^{\prime} \geq 0$ and $E^{\prime} \leq 0$ since (2.6)),

$$
\begin{aligned}
& \mathcal{F}^{\prime}(t)=\frac{\varepsilon}{E(0)}\left(\frac{E(t)}{\tilde{h}(t)+1}\right)^{\prime} G^{\prime \prime}\left(\frac{\varepsilon E(t)}{E(0)(\tilde{h}(t)+1)}\right) L(t)+G^{\prime}\left(\frac{\varepsilon E(t)}{E(0)(\tilde{h}(t)+1)}\right) \\
& \times L^{\prime}(t) \leq-c E(t) G^{\prime}\left(\frac{\varepsilon E(t)}{E(0)(\tilde{h}(t)+1)}\right)+\hat{c} h(t) G^{\prime}\left(\frac{\varepsilon E(t)}{E(0)(\tilde{h}(t)+1)}\right) \\
& +\frac{\hat{c}(\tilde{h}(t)+1)}{\epsilon_{0}} G^{\prime}\left(\frac{\varepsilon E(t)}{E(0)(\tilde{h}(t)+1)}\right) G^{-1}\left(\frac{\epsilon_{0} I(t)}{\xi(t)(\tilde{h}(t)+1)}\right) .
\end{aligned}
$$


Let $G^{*}$ be the convex conjugate of $G$ in the sense of Young (see [2]), then

$$
G^{*}(s)=s\left(G^{\prime}\right)^{-1}(s)-G\left[\left(G^{\prime}\right)^{-1}(s)\right] \leq s\left(G^{\prime}\right)^{-1}(s), \quad \forall s \in \mathbb{R}_{+}
$$

and $G^{*}$ satisfies the following generalized Young inequality:

$$
A B \leq G(A)+G^{*}(B), \quad \forall A, B \in \mathbb{R}_{+} .
$$

So, with $A=G^{-1}\left(\frac{\epsilon_{0} I(t)}{\xi(t)(\tilde{h}(t)+1)}\right)$ and $B=G^{\prime}\left(\frac{\varepsilon E(t)}{E(0)(\tilde{h}(t)+1)}\right)$ and using (4.12)(4.13), we arrive at, for any $t \in \mathbb{R}_{+}$,

$$
\begin{aligned}
& \mathcal{F}^{\prime}(t) \leq-c E(t) G^{\prime}\left(\frac{\varepsilon E(t)}{E(0)(\tilde{h}(t)+1)}\right)+\hat{c} \frac{I(t)}{\xi(t)}+\hat{c} h(t) G^{\prime}\left(\frac{\varepsilon E(t)}{E(0)(\tilde{h}(t)+1)}\right) \\
& +\frac{\hat{c}(\tilde{h}(t)+1)}{\epsilon_{0}} G^{*}\left(G^{\prime}\left(\frac{\varepsilon E(t)}{E(0)(\tilde{h}(t)+1)}\right)\right) \leq-c E(t) G^{\prime}\left(\frac{\varepsilon E(t)}{E(0)(\tilde{h}(t)+1)}\right) \\
& +\frac{\hat{c}}{\epsilon_{0}} \frac{\varepsilon E(t)}{E(0)} G^{\prime}\left(\frac{\varepsilon E(t)}{E(0)(\tilde{h}(t)+1)}\right)+\hat{c} \frac{I(t)}{\xi(t)}+\hat{c} h(t) G^{\prime}\left(\frac{\varepsilon E(t)}{E(0)(\tilde{h}(t)+1)}\right) .
\end{aligned}
$$

By choosing $0<\varepsilon<\frac{c \epsilon_{0} E(0)}{\hat{c}}$, multiplying the above inequality by $\xi(t)$ and recalling the definition of $G_{0}$ in (4.1) and (3.9), we obtain, for any $t \in \mathbb{R}_{+}$,

$$
\begin{aligned}
\xi(t) \mathcal{F}^{\prime}(t) \leq & -c \xi(t)(\tilde{h}(t)+1) G_{0}\left(\frac{\varepsilon E(t)}{E(0)(\tilde{h}(t)+1)}\right) \\
& -\hat{c}\left(\delta_{0} E^{\prime}(t)+\left(1-\delta_{0}\right) E_{*}^{\prime}(t)\right)+\hat{c} \xi(t) h(t) G^{\prime}\left(\frac{\varepsilon E(t)}{E(0)(\tilde{h}(t)+1)}\right) .
\end{aligned}
$$

Let $\mathcal{F}_{1}=\xi \mathcal{F}+\hat{c}\left(\delta_{0} E+\left(1-\delta_{0}\right) E_{*}\right)$. From (3.2) and (4.11), and noting that $0 \leq G^{\prime}\left(\frac{\varepsilon E(t)}{E(0)(\tilde{h}(t)+1)}\right) \leq G^{\prime}(\varepsilon)$ and $0 \leq \xi(t) \leq \xi(0)$, we deduce that

$$
\mathcal{F}_{1} \sim E \quad \text { for } \quad(1.1), \quad \text { and } \quad \mathcal{F}_{1} \geq \hat{c} E_{*} \geq c E \quad \text { for } \quad(1.2),
$$

and because $\xi$ is nonincreasing, (4.14) implies that, for any $t \in \mathbb{R}_{+}$,

$$
\begin{aligned}
\mathcal{F}_{1}^{\prime}(t) & \leq-c \xi(t)(\tilde{h}(t)+1) G_{0}(\varepsilon E(t) /(E(0)(\tilde{h}(t)+1))) \\
& +\hat{c} \xi(t) h(t) G^{\prime}(\varepsilon E(t)(E(0)(\tilde{h}(t)+1))) .
\end{aligned}
$$

Let $d>0$. Using the definition of $G_{0}$ and $G_{2}$ in (4.1) and the general Young inequality (4.13) for the last term in (4.16) with $A=G^{\prime}\left(\frac{\varepsilon E(t)}{E(0)(\tilde{h}(t)+1)}\right)$, 
$B=\hat{c} h(t) / d(\tilde{h}(t)+1)$ and $G_{2}$ instead of $G\left(G_{2}\right.$ is convex thanks to (A2)), we have, for any $t \in \mathbb{R}_{+}$,

$$
\begin{aligned}
& \hat{c} h(t) G^{\prime}\left(\frac{\varepsilon E(t)}{E(0)(\tilde{h}(t)+1)}\right)=d(\tilde{h}(t)+1)\left(\frac{\hat{c} h(t)}{d(\tilde{h}(t)+1)}\right) G^{\prime}\left(\frac{\varepsilon E(t)}{E(0)(\tilde{h}(t)+1)}\right) \\
& \leq d(\tilde{h}(t)+1)\left[G_{2}\left(G^{\prime}\left(\frac{\varepsilon E(t)}{E(0)(\tilde{h}(t)+1)}\right)+G_{2}^{*}\left(\frac{\hat{c} h(t)}{d(\tilde{h}(t)+1)}\right)\right]\right. \\
& \leq d(\tilde{h}(t)+1)\left[\frac{\varepsilon E(t)}{E(0)(\tilde{h}(t)+1)} G^{\prime}\left(\frac{\varepsilon E(t)}{E(0)(\tilde{h}(t)+1)}\right)+G_{2}^{*}\left(\frac{\hat{c} h(t)}{d(\tilde{h}(t)+1)}\right)\right] \\
& \leq d(\tilde{h}(t)+1)\left[G_{0}\left(\frac{\varepsilon E(t)}{E(0)(\tilde{h}(t)+1)}\right)+G_{2}^{*}\left(\frac{\hat{c} h(t)}{d(\tilde{h}(t)+1)}\right)\right] .
\end{aligned}
$$

Combining (4.16) and (4.17) and choosing $d$ small enough, we arrive at, for any $t \in \mathbb{R}_{+}$,

$$
\mathcal{F}_{1}^{\prime}(t) \leq \xi(t)(\tilde{h}(t)+1)\left[-c G_{0}\left(\frac{\varepsilon E(t)}{E(0)(\tilde{h}(t)+1)}\right)+\hat{c} G_{2}^{*}\left(\frac{\hat{c} h(t)}{\tilde{h}(t)+1}\right)\right] .
$$

Now, we distiguish the cases (1.1) and (1.2).

Case (1.1). Taking into account the first property in (4.15), we have, for some $d_{0}>0$,

$$
G_{0}\left(\frac{\varepsilon E(t)}{E(0)(\tilde{h}(t)+1)}\right) \geq G_{0}\left(\frac{d_{0} \mathcal{F}_{1}(t)}{\tilde{h}(t)+1}\right), \quad \forall t \in \mathbb{R}_{+} .
$$

Then, puting $\mathcal{F}_{2}=\frac{d_{0}}{\tilde{h}+1} \mathcal{F}_{1}$, we see that (4.18) implies, for positive constants $c_{1}$ and $c_{2}$,

$$
\mathcal{F}_{2}^{\prime}(t) \leq-c_{1} \xi(t) G_{0}\left(\mathcal{F}_{2}(t)\right)+c_{2} \xi(t) G_{2}^{*}\left(\frac{c_{2} h(t)}{\tilde{h}(t)+1}\right), \quad \forall t \in \mathbb{R}_{+},
$$

since $d_{0} /(\tilde{h}+1)$ is nonincreasing. The first property in (4.15) implies that there exists $b_{0}>0$ such that $\mathcal{F}_{2} \geq b_{0} /(\tilde{h}+1) E$. Let $t \in \mathbb{R}_{+}$and $\alpha$ satisfying (4.3) and (4.4). If $b_{0} /(\tilde{h}(t)+1) E(t) \leq 2 \gamma(t) / \alpha(t)$, then

$$
E(t) \leq \frac{2}{b_{0}}(\tilde{h}(t)+1) \frac{\gamma(t)}{\alpha(t)}
$$

If $\frac{b_{0}}{\hat{h}(t)+1} E(t)>2 \frac{\gamma(t)}{\alpha(t)}$, then $\frac{b_{0}}{\hat{h}(s)+1} E(s)>2 \frac{\gamma(t)}{\alpha(t)}$, for any $0 \leq s \leq t$, since $\frac{E}{\tilde{h}+1}$ is nonincreasing. Therefore $\mathcal{F}_{2}(s)>2 \frac{\gamma(t)}{\alpha(t)}$, for any $0 \leq s \leq t$. Using the fact that $G_{0}$ is convex, $G_{0}(0)=0$ (assumption (A2)) and $0<\alpha \leq 1$, we have, for any 
$0 \leq s \leq t$ and $0<\epsilon_{1} \leq 1$

$$
\begin{aligned}
& G_{0}\left(\epsilon_{1} \alpha(s) \mathcal{F}_{2}(s)-\epsilon_{1} \gamma(s)\right) \leq \epsilon_{1} \alpha(s) G_{0}\left(\mathcal{F}_{2}(s)-\gamma(s) / \alpha(s)\right) \\
& \leq \epsilon_{1} \alpha(s) \mathcal{F}_{2}(s) G^{\prime}\left(\mathcal{F}_{2}(s)-\frac{\gamma(s)}{\alpha(s)}\right)-\epsilon_{1} \alpha(s) \frac{\gamma(s)}{\alpha(s)} G^{\prime}\left(\mathcal{F}_{2}(s)-\frac{\gamma(s)}{\alpha(s)}\right) \\
& \leq \epsilon_{1} \alpha(s) \mathcal{F}_{2}(s) G^{\prime}\left(\mathcal{F}_{2}(s)\right)-\epsilon_{1} \alpha(s) \frac{\gamma(s)}{\alpha(s)} G^{\prime}\left(\frac{\gamma(s)}{\alpha(s)}\right),
\end{aligned}
$$

thus, we put $\mathcal{F}_{3}=\epsilon_{1} \alpha \mathcal{F}_{2}-\epsilon_{1} \gamma$ with $0<\epsilon_{1} \leq 1$ such that $\mathcal{F}_{3}(0) \leq 1$, we get

$$
G_{0}\left(\mathcal{F}_{3}(s)\right) \leq \epsilon_{1} \alpha(s) G_{0}\left(\mathcal{F}_{2}(s)\right)-\epsilon_{1} \alpha(s) G_{0}(\gamma(s) / \alpha(s)), \quad \forall 0 \leq s \leq t .
$$

Because $\alpha^{\prime} \leq 0$ (from (4.3)), we deduce from (4.19) and (4.20) that

$$
\mathcal{F}_{3}^{\prime}(s) \leq-c_{1} \xi(s) G_{0}\left(\mathcal{F}_{3}(s)\right)+\psi(s), \quad \forall 0 \leq s \leq t,
$$

where

$$
\psi(s)=\epsilon_{1} \xi(s) \alpha(s)\left(c_{2} G_{2}^{*}\left(\frac{c_{2} h(s)}{\tilde{h}(s)+1}\right)-c_{1} G_{0}\left(\frac{\gamma(s)}{\alpha(s)}\right)\right)-\epsilon_{1} \gamma^{\prime}(s) .
$$

From the definition of $G_{1}$ and $\gamma$ in (4.1) and (4.5), we have

$$
G_{1}(\gamma(s))=c_{1} \int_{0}^{s} \xi(\tau) d \tau \quad \Longrightarrow \quad \gamma^{\prime}(s)=-c_{1} \xi(s) G_{0}(\gamma(s)), \quad \forall 0 \leq s \leq t,
$$

which leads to $\psi \leq 0$ according to (4.4). Then we get from (4.21) that

$$
\left(G_{1}\left(\mathcal{F}_{3}(s)\right)\right)^{\prime} \geq c_{1} \xi(s), \quad \forall 0 \leq s \leq t .
$$

By integrating (4.22) over $[0, t]$, we get

$$
G_{1}\left(\mathcal{F}_{3}(t)\right) \geq c_{1} \int_{0}^{t} \xi(s) d s+G_{1}\left(\mathcal{F}_{3}(0)\right) .
$$

But $G_{1}$ is decreasing, $\mathcal{F}_{3}(0) \leq 1$ and $G_{1}(1)=0$, then

$$
\mathcal{F}_{3}(t) \leq G_{1}^{-1}\left(c_{1} \int_{0}^{t} \xi(s) d s\right)=\gamma(t) .
$$

Therefore, by definition of $\mathcal{F}_{3}$ and $\mathcal{F}_{2}$, we get

$$
\mathcal{F}_{2}(t) \leq \frac{\epsilon_{1}+1}{\epsilon_{1}} \frac{\gamma(t)}{\alpha(t)} \quad \text { and } \quad \mathcal{F}_{1}(t) \leq \frac{\epsilon_{1}+1}{\epsilon_{1} d_{0}}(\tilde{h}(t)+1) \frac{\gamma(t)}{\alpha(t)}
$$

But $\mathcal{F}_{1} \sim E$ implies that $E \leq b_{1} \mathcal{F}_{1}$, for some $b_{1}>0$, then

$$
E(t) \leq \frac{\left(\epsilon_{1}+1\right) b_{1}}{\epsilon_{1} d_{0}}(\tilde{h}(t)+1) \frac{\gamma(t)}{\alpha(t)}
$$

Finally, we deduce that (4.7) holds, for any $t \in \mathbb{R}_{+}$, with $c_{3}=\max \left\{\frac{2}{b_{0}}, \frac{\left(\epsilon_{1}+1\right) b_{1}}{\epsilon_{1} d_{0}}\right\}$. 
Case (1.2). Because $\frac{1}{\tilde{h}+1}$ is nonicreasing and $\mathcal{F}_{1}$ is nonegative, then (4.18) leads to

$$
c \xi(s) G_{0}\left(\frac{\varepsilon E(s)}{E(0)(\tilde{h}(s)+1)}\right) \leq-\left(\frac{\mathcal{F}_{1}(s)}{\tilde{h}(s)+1}\right)^{\prime}+\hat{c} \xi(s) G_{2}^{*}\left(\frac{\hat{c} h(s)}{\tilde{h}(s)+1}\right), \forall s \in \mathbb{R}_{+} .
$$

So, noting that $G_{0}\left(\frac{\varepsilon E}{E(0)(\tilde{h}+1)}\right)$ is nonincreasing and integrating the above inequality over $[0, t]$, we get

$$
\begin{aligned}
& c G_{0}\left(\frac{\varepsilon E(t)}{E(0)(\tilde{h}(t)+1)}\right) \int_{0}^{t} \xi(s) d s \leq c \int_{0}^{t} \xi(s) G_{0}\left(\frac{\varepsilon E(s)}{E(0)(\tilde{h}(s)+1)}\right) d s \\
& \leq \mathcal{F}_{1}(0)-\frac{\mathcal{F}_{1}(t)}{\tilde{h}(t)+1}+\hat{c} \int_{0}^{t} \xi(s) G_{2}^{*}\left(\frac{\hat{c} h(s)}{\tilde{h}(s)+1}\right) d s \\
& \leq \mathcal{F}_{1}(0)+\hat{c} \int_{0}^{t} \xi(s) G_{2}^{*}\left(\frac{\hat{c} h(s)}{\tilde{h}(s)+1}\right) d s, \quad \forall t \in \mathbb{R}_{+},
\end{aligned}
$$

which leads to (4.8) with $c_{1}=\max \left\{\frac{E(0)}{\varepsilon}, \frac{\mathcal{F}_{1}(0)}{c}, \frac{\hat{c}}{c}, \hat{c}\right\}$. The proof of Theorem 2 is now completed.

Remark 3. 1. From (4.4), we see that

$$
\frac{\gamma(t)}{\alpha(t)} \geq G_{0}^{-1}\left(\frac{c_{2}}{c_{1}} G_{2}^{*}\left(\frac{c_{2} h(t)}{\tilde{h}(t)+1}\right)\right), \quad \forall t \in \mathbb{R}_{+} .
$$

Consequently, the decay rate at infinity of $E$ given by (4.7) can not be better than the one of the function

$$
t \mapsto c_{3}(\tilde{h}(t)+1) G_{0}^{-1}\left(\frac{c_{2}}{c_{1}} G_{2}^{*}\left(\frac{c_{2} h(t)}{\tilde{h}(t)+1}\right)\right) .
$$

2. The class of functions $\alpha$ satisfying (4.3) and (4.4) is not empty; it contains at least the function $\alpha=d \gamma$, with $0<d \leq 1$ small enough. Indeed, (4.3) is satisfied (since (4.1) and (4.5)). On the other hand, we have $\frac{h}{\tilde{h}+1}$ is nonincreasing, $0<\gamma \leq 1$, and $G^{\prime}$ and $G_{2}^{*}$ are increasing, then (4.4) is satisfied if

$$
c_{2} G_{2}^{*}\left(c_{2} h(0)\right) \leq \frac{c_{1}}{d}\left(G^{\prime}\left(\frac{1}{d}\right)-G^{\prime}(1)\right)
$$

which holds, for $0<d \leq 1$ small enough, since $\lim _{t \rightarrow+\infty} G^{\prime}(t)=+\infty$. But with the choice $\alpha=d \gamma,(4.7)$ does not lead to any stability estimate. The idea is to choose $\alpha$ satisfying (4.3) and (4.4) such that (4.7) gives the best possible decay rate for $E$. On the other hand, the decay rate given by (4.8) depends on the one of $s \mapsto\left\|\nabla u_{0}(s)\right\|_{2}$. See the two examples below. 
Example 1. Let us consider the following class of $g$ and $u_{0}$ :

$$
g(s)=a(s+1)^{-q} \text { and } m_{1}(s+1)^{r} \leq 1+\left\|\nabla u_{0}(s)\right\|_{2}^{2} \leq m_{0}(s+1)^{r},
$$

where $m_{0}, m_{1}>0, q>1,0<a<q-1$ for (1.1), $0<a<\frac{q-1}{c_{0}}$ for (1.2) (then (A1) is satisfied, for any $\beta_{0} \geq q$ ) and $0 \leq r<q-1$ (so $\eta_{0} \in L_{g}^{1}$ ). The assumption (A2) holds with $\xi(s)=q a^{\frac{-1}{q}}$ and $G(s)=s^{\frac{q+1}{q}}$. Then, there exist positive constants $d_{i}(i=0, \ldots, 4)$ depending only on $a, q$ and $c_{1}$ such that (using (4.6))

$$
G_{0}(s)=d_{0} s^{\frac{q+1}{q}}, \quad G_{1}(s)=d_{1}\left(s^{\frac{-1}{q}}-1\right), \quad G_{2}(s)=d_{2} s^{q+1}, \quad G_{2}^{*}(s)=d_{3} s^{\frac{q+1}{q}}
$$

and $\gamma(s)=\left(d_{4} s+1\right)^{-q}$. On the other hand, we have

$$
\begin{aligned}
& d_{5}(t+1)^{-(q-r-1)} \leq h(t) \leq d_{6}(t+1)^{-(q-r-1)}, \quad \forall t \in \mathbb{R}_{+}, \\
& \tilde{h}(t)+1 \geq d_{7}\left\{\begin{array}{ll}
\ln (t+1)+1 & \text { if } q-r=2, \\
1 & \text { if } q-r>2, \\
(t+1)^{-q+r+2} & \text { if } 1<q-r<2,
\end{array} \quad \forall t \in \mathbb{R}_{+}\right.
\end{aligned}
$$

and

$$
\tilde{h}(t)+1 \leq d_{8}\left\{\begin{array}{ll}
\ln (t+1)+1 & \text { if } q-r=2, \\
1 & \text { if } q-r>2, \\
(t+1)^{-q+r+2} & \text { if } 1<q-r<2,
\end{array} \quad \forall t \in \mathbb{R}_{+},\right.
$$

for some constant $d_{5}, d_{6}, d_{7}, d_{8}>0$ (depending only on $a, q, m_{0}, m_{1}$ and $r$ ). Condition (4.4) is satisfied if

$$
\frac{(t+1)^{q} h(t)}{\tilde{h}(t)+1} \alpha(t) \leq d_{9}\left(1-(\alpha(t))^{\frac{1}{q}}\right)^{\frac{q}{q+1}}, \quad \forall t \in \mathbb{R}_{+},
$$

where $d_{9}>0$ depending only on $a, q, c_{1}$ and $c_{2}$. Choosing $\alpha(t)=\lambda(t+1)^{-p}$ with $0<\lambda \leq 1, p=r+1$ if $q-r \geq 2$, and $p=q-1$ if $1<q-r<2$, so (4.3) is valid. Moreover, using (4.24) and (4.25), we see that (4.27) is satisfied if $0<\lambda \leq 1$ is small enough, and then (4.4) is satisfied. Hence (4.7) and (4.26) imply that, for any $t \in \mathbb{R}_{+}$,

$$
E(t) \leq \begin{cases}c_{3}(\ln (t+1)+1)(t+1)^{-1} & \text { if } q-r=2, \\ c_{3}(t+1)^{-(q-r-1)} & \text { if } q-r>2 \text { or } 1<q-r<2 .\end{cases}
$$

The estimate (4.28) leads to

$$
\lim _{t \rightarrow+\infty} E(t)=0
$$

even if $s \mapsto\left\|\nabla u_{0}(s)\right\|_{2}$ is not bounded (that is $0<r<q-1$ in (4.23)). The estimate (4.28) extends and improves the decay rate $(t+1)^{-\tilde{p}}$ (for some $0<\tilde{p}$ small enough) obtained in [19] for $q>2$. 
If, for example, $s \mapsto\left\|\nabla u_{0}(s)\right\|_{2}$ is bounded; that is $r=0$ in (4.23) (as it was assumed in $[15,17,40])$, then $(4.28)$ holds with $r=0$, which gives a better decay rate than the ones $(t+1)^{-\tilde{p}}$ (for any $0<\tilde{p}<\frac{q-1}{2}$ ), $(t+1)^{-\tilde{p}}$ (for some $0<\tilde{p}$ small enough) and $(t+1)^{-\frac{q^{2}-q-1}{q}}$ obtained in $[15,17,40]$, respectively.

Using (4.24) and (4.25), we see that

$$
\int_{0}^{+\infty} \xi(s) G_{2}^{*}\left(\frac{c_{1} h(s)}{\tilde{h}(s)+1}\right) d s<+\infty,
$$

then (4.8) and (4.26) imply that the solution of (1.2) satisfies

$$
E(t) \leq\left\{\begin{array}{ll}
c_{1}(\ln (t+1)+1) t^{-\frac{q}{q+1}} & \text { if } q-r=2, \\
c_{1} t^{-\frac{q}{q+1}} & \text { if } q-r>2, \\
c_{1} t^{-\left(\frac{q}{q+1}+q-r-2\right)} & \text { if } 1<q-r<2,
\end{array}, \quad \forall t \in \mathbb{R}_{+}^{*} .\right.
$$

If $q-r \geq 2$ or $\frac{1}{2}\left(r+\sqrt{r^{2}+4(r+2)}\right)<q<r+2$, the estimate (4.30) in case $q>\sqrt{2}$ implies (4.29) even if $s \mapsto\left\|\nabla u_{0}(s)\right\|_{2}$ is not bounded.

If $s \mapsto\left\|\nabla u_{0}(s)\right\|_{2}$ is bounded $(r=0$ in (4.23)) as it was assumed in $[15,17$, 40], we get (4.30) with $r=0$. In this case, we get a better decay rate than the one $(t+1)^{-\tilde{p}}$ (for any $0<\tilde{p}<\frac{q-1}{q+1}$ ) obtained in [15].

Example 2.

Let us consider the second example of the following class of $g$ and $u_{0}$ :

$$
\begin{aligned}
& g(s)=a(s+2)^{-1}(\ln (s+2))^{-q}, \\
& m_{1}(\ln (s+2))^{r} \leq 1+\left\|\nabla u_{0}(s)\right\|_{2}^{2} \leq m_{0}(\ln (s+2))^{r}, \quad \forall s \in \mathbb{R}_{+},
\end{aligned}
$$

where $m_{0}, m_{1}>0, q>1,0<a<(q-1)(\ln 2)^{q-1}$ in case (1.1), $0<a<$ $\frac{q-1}{c_{0}}(\ln 2)^{q-1}$ in case $(1.2)$ (so (A1) holds, for any $\beta_{0} \geq \frac{1}{2}\left(1+\frac{q}{\ln 2}\right)$ ) and $0 \leq r<q-1$ (then $\eta_{0} \in L_{g}^{1}$ ). The assumption (A2) holds with $\xi(s)=d_{0}$ and $G(s)=s^{2}$, where $d_{0}>0$ depends only on $a$ and $q$. Then, for $d_{1}>0$ (depending only on $d_{0}$ and $c_{1}$ ),

$$
G_{0}(s)=2 s^{2}, \quad G_{2}(s)=G_{2}^{*}(s)=\frac{1}{2} s^{2}, \quad G_{1}(s)=\frac{1}{2}\left(\frac{1}{s}-1\right)
$$

and $\gamma(s)=\left(d_{1} s+1\right)^{-1}$. Similarily to the first example, (4.32) implies that, for some $d_{2}, d_{3}, d_{4}, d_{5}>0$ (depending only on $a, q, m_{0}, m_{1}$ and $r$ ) and for any $t \in \mathbb{R}_{+}$,

$$
\begin{aligned}
& d_{2}(\ln (t+2))^{-(q-r-1)} \leq h(t) \leq d_{3}(\ln (t+2))^{-(q-r-1)} \\
& d_{4}(\ln (t+2))^{-(q-r-1)}(t+1) \leq \tilde{h}(t)+1 \leq d_{5}(\ln (t+2))^{-(q-r-1)}(t+1) .
\end{aligned}
$$

Using (4.33) and (4.34), we see that condition (4.4) is satisfied if

$$
\alpha(t) \leq d_{6}(1-\alpha(t))^{\frac{1}{2}}, \quad \forall t \in \mathbb{R}_{+},
$$


where $d_{6}>0$ (depending only on $a, q, r, m_{0}, m_{1}, c_{1}$ and $c_{2}$ ). By choosing $\alpha(t)=\lambda$ with $0<\lambda \leq 1$ small enough, we get (4.3) and (4.35). Then we deduce from (4.7) and (4.34) that the solution of (1.1) satisfies

$$
E(t) \leq c_{3}(\ln (t+2))^{-(q-r-1)}, \quad \forall t \in \mathbb{R}_{+},
$$

which imply (4.29) even if $s \mapsto\left\|\nabla u_{0}(s)\right\|_{2}$ is not bounded $(0<r<q-1$ in (4.32)). However, (4.8) does not give in case (4.31) any decay estimate for the solution of (1.2).

If $s \mapsto\left\|\nabla u_{0}(s)\right\|_{2}$ is bounded $(r=0$ in (4.32)) as it was assumed in [15,17], then the decay rate given in (4.36) with $r=0$ is better than the one $(\ln (t+2))^{-\tilde{p}}$ (for some $0<\tilde{p}$ small enough) obtained in [17], and it is little better than the one $(\ln (t+2))^{-\tilde{p}}$ (for any $\left.0<\tilde{p}<q-1\right)$ given in [15].

\section{Applications}

1. Our systems (1.1) and (1.2) are particular problems that fall in the framework of the abstract system (1.13) (considered in [15]) in a Hilbert space $H$ with a norm denoted by $\|\cdot\|$, where $A: D(A) \rightarrow H$ and $B: D(B) \rightarrow H$ are self-adjoint linear positive definite operators with domains $D(A) \subset D(B) \subset H$ such that the embeddings are dense and compact, and there exist $a_{0}, a_{1}>0$ satisfying

$$
\begin{aligned}
& a_{1}\|v\|^{2} \leq\left\|B^{\frac{1}{2}} v\right\|^{2} \leq a_{0}\left\|A^{\frac{1}{2}} v\right\|^{2}, \quad \forall v \in D\left(A^{\frac{1}{2}}\right), \\
& 0<g_{0}<1 / a_{0} .
\end{aligned}
$$

Systems (1.1) and (1.2) are included, respectively, in the following two distinguished cases considered in [15]:

$$
\begin{aligned}
& \exists a_{2}>0:\left\|A^{\frac{1}{2}} v\right\|^{2} \leq a_{2}\left\|B^{\frac{1}{2}} v\right\|^{2}, \quad \forall v \in D\left(A^{\frac{1}{2}}\right), \\
& \exists a_{2}>0:\left\|A^{\frac{1}{2}} v\right\|^{2} \leq a_{2}\left\|A^{\frac{1}{2}} B^{\frac{1}{2}} v\right\|^{2}, \quad \forall v \in D\left(A^{\frac{1}{2}} B^{\frac{1}{2}}\right) .
\end{aligned}
$$

System (1.1) corresponds to the case (4.39) with $H=L^{2}(\Omega), A=B=$ $-\Delta, D\left(A^{\frac{1}{2}}\right)=D\left(B^{\frac{1}{2}}\right)=H_{0}^{1}(\Omega)$ and $D(A)=D(B)=H^{2}(\Omega) \cap H_{0}^{1}(\Omega)$. And system (1.2) corresponds to the case (4.40) with $H=L^{2}(\Omega), A=$ $-\Delta, B=I d, D\left(A^{\frac{1}{2}}\right)=H_{0}^{1}(\Omega), D(A)=H^{2}(\Omega) \cap H_{0}^{1}(\Omega)$ and $D(B)=$ $D\left(B^{\frac{1}{2}}\right)=L^{2}(\Omega)$. Our stability results can be extended to the abstract system (1.13) under assumptions [(A1), (A2), (4.37), (4.38) (instead of the second condition in (2.1))] and [(4.39) or (4.40)], so the estimates (4.7) and (4.8) hold in cases (4.39) and (4.40), respectively, where $\nabla$ in (4.2) is replaced by $A^{\frac{1}{2}}$ and $A^{\frac{1}{2}} B^{\frac{1}{2}}$ in cases (4.39) and (4.40), respectively. So we improve the results of [15] by droping the boundedness condition (1.14) on $u_{0}$ and obtaining a better decay rate.

2. The aprroach presented in this paper can be also applied to (1.16) and (1.20) in order to improve the decay rate of solutions obtained in $[16,19]$, and drop the boundedness condition (1.17) on $u_{0}$. 


\section{Acknowledgements}

This work was initiated during the visit of the athor to KFUPM, Saudi Arabia, in December 2018. The author is grateful for the support and facilities provided by KFUPM. The author would like to express his gratitude to the anonymous referees for very careful reading and punctual suggestions.

\section{References}

[1] J.A. Appleby, M. Fabrizio, B. Lazzari and D.W. Reynolds. On exponential asymptotic stability in linear viscoelasticity. Mathematical Models and Methods in Applied Sciences, 16(10):1677-1694, 2006. https://doi.org/10.1142/S0218202506001674.

[2] V.I. Arnold. Mathematical methods of classical mechanics. Springer Science \& Business Media, 2013.

[3] S. Berrimi and S.A. Messaoudi. Exponential decay of solutions to a viscoelastic equation with nonlinear localized damping. Electronic Journal of Differential Equations, 88:1-10, 2004.

[4] M. Cavalcanti, V.N. Domingos Cavalcanti and J. Ferreira. Existence and uniform decay for a non-linear viscoelastic equation with strong damping. Mathematical Methods in the Applied Sciences, 24(14):1043-1053, 2001. https://doi.org/10.1002/mma.250.

[5] M. Cavalcanti and A. Guesmia. General decay rates of solutions to a nonlinear wave equation with boundary condition of memory type. Differential and Integral Equations, 18:583-600, 2005.

[6] M.M. Cavalcanti, V.N. Domingos Cavalcanti and J.A. Soriano. Exponential decay for the solution of semilinear viscoelastic wave equations with localized damping. Electronic Journal of Differential Equations, 44:1-14, 2002.

[7] M.M. Cavalcanti and H.P. Oquendo. Frictional versus viscoelastic damping in a semilinear wave equation. SIAM Journal on Control and Optimization, 42(4):1310-1324, 2003. https://doi.org/10.1137/S0363012902408010.

[8] R. Christensen. Theory of viscoelasticity: an introduction. Elsevier, 2012.

[9] M. Conti and V. Pata. Weakly dissipative semilinear equations of viscoelasticity. Communications on Pure and Applied Analysis, 4(4):705-720, 2005. https://doi.org/10.3934/cpaa.2005.4.705.

[10] C.M. Dafermos. An abstract Volterra equation with applications to linear viscoelasticity. Journal of Differential Equations, 7(3):554-569, 1970. https://doi.org/10.1016/0022-0396(70)90101-4,.

[11] C.M. Dafermos. Asymptotic stability in viscoelasticity. Archive for Rational Mechanics and Analysis, 37:297-308, 1970. https://doi.org/10.1007/BF00251609.

[12] G. Dassios and F. Zafiropoulos. Equipartition of energy in linearized 3d viscoelasticity. Quarterly of Applied Mathematics, 48:715-730, 1990. https://doi.org/10.1090/qam/1079915.

[13] C. Giorgi, J.E. Muñoz Rivera and V. Pata. Global attractors for a semilinear hyperbolic equation in viscoelasticity. Journal of Mathematical Analysis and Applications, 260(1):83-99, 2001. https://doi.org/10.1006/jmaa.2001.7437. 
[14] A. Guesmia. Stabilisation de l'équation des ondes avec conditions aux limites de type mémoire. Afrika Mathematika, 10:14-25, 1999.

[15] A. Guesmia. Asymptotic stability of abstract dissipative systems with infinite memory. Journal of Mathematical Analysis and Applications, 382(2):748-760, 2011. https://doi.org/10.1016/j.jmaa.2011.04.079.

[16] A. Guesmia. Asymptotic behavior for coupled abstract evolution equations with one infinite memory. Applicable Analysis, 94:184-217, 2015. https://doi.org/10.1080/00036811.2014.890708.

[17] A. Guesmia and S. Messaoudi. A new approach to the stability of an abstract system in the presence of infinite history. Journal of Mathematical Analysis and Applications, 416:212-228, 2014. https://doi.org/10.1016/j.jmaa.2014.02.030.

[18] A. Guesmia and S.A. Messaoudi. A general decay result for a viscoelastic equation in the presence of past and finite history memories. Nonlinear Analysis: Real World Applications, 13(1):476-485, 2012. https://doi.org/10.1016/j.nonrwa.2011.08.004.

[19] A. Guesmia and N. Tatar. Some well-posedness and stability results for abstract hyperbolic equations with infinite memory and distributed time delay. Communications on Pure and Applied Analysis, 14(2):457-491, 2015. https://doi.org/10.3934/cpaa.2015.14.457.

[20] J.H. Hao and F. Wang. General decay rate for weak viscoelastic wave equation with Balakrishnan-Taylor damping and time-varying delay. Computers and Mathematics with Applications, 78(9):2632-2640, 2019. https://doi.org/10.1016/j.camwa.2019.04.010.

[21] J.H. Hao and P. Wang. General stability result of abstract thermoelastic system with infinite memory. Bulletin of the Malaysian Mathematical Sciences Society, 42:2549-2567, 2019. https://doi.org/10.1007/s40840-018-0615-z.

[22] J.H. Hao and J. Wei. Intrinsic decay rate estimates for abstract wave equation with memory. Mathematical Methods in the Applied Sciences, 40:5131-5140, 2017. https://doi.org/10.1002/mma.4375.

[23] J. Hashim Hassan and S.A. Messaoudi. General decay rate for a class of weakly dissipative second-order systems with memory. Mathematical Methods in the Applied Sciences, 42:2842-2853, 2019. https://doi.org/10.1002/mma.5554.

[24] W.J. Hrusa. Global existence and asymptotic stability for a semilinear hyperbolic Volterra equation with large initial data. SIAM Journal on Mathematical Analysis, 16(1):110-134, 1985. https://doi.org//10.1137/0516007.

[25] K.P. Jin, J. Liang and T.J. Xiao. Coupled second order evolution equations with fading memory: optimal energy decay rate. Journal of Differential Equations, 257(5):1501-1528, 2014. https://doi.org//10.1016/j.jde.2014.05.018.

[26] K.P. Jin, J. Liang and T.J. Xiao. Asymptotic behavior of coupled systems of second order abstract evolution equations with one infinite memory. Journal of Mathematical Analysis and Applications, 475(1):554-575, 2019. https://doi.org/10.1016/j.jmaa.2019.02.055.

[27] F. Li, Z. Zhao and Y. Chen. Global existence uniqueness and decay estimates for nonlinear viscoelastic wave equation with boundary dissipation. Nonlinear Analysis: Real World Applications, 12(3):1759-1773, 2011. https://doi.org/10.1016/j.nonrwa.2010.11.009. 
[28] S.A. Messaoudi. General decay of solutions of a viscoelastic equation. Journal of Mathematical Analysis and Applications, 341(2):1457-1467, 2008. https://doi.org/10.1016/j.jmaa.2007.11.048.

[29] S.A. Messaoudi. General decay of the solution energy in a viscoelastic equation with a nonlinear source. Nonlinear Analysis: Theory, Methods and Applications, 69:2589-2598, 2008. https://doi.org/10.1016/j.na.2007.08.035.

[30] S.A. Messaoudi and W. Al-Khulaifi. General and optimal decay for a quasilinear viscoelastic equation. Applied Mathematics Letters, 66:16-22, 2017. https://doi.org/10.1016/j.aml.2016.11.002.

[31] S.A. Messaoudi and N. Tatar. Global existence and asymptotic behavior for nonlinear viscoelastic problem. Mathematical Sciences Research Journal, 7:136149, 2003. https://doi.org/10.1002/mma.804.

[32] S.A. Messaoudi and N. Tatar. Global existence and uniform stability of solutions for a quasilinear viscoelastic problem. Mathematical Methods in the Applied Sciences, 30:665-680, 2007.

[33] M.I. Mustafa. Optimal decay rates for the viscoelastic wave equation. Mathematical Methods in the Applied Sciences, 41(1):192-204, 2018. https://doi.org/10.1002/mma.4604.

[34] M.I. Mustafa and S.A. Messaoudi. General stability result for viscoelastic wave equations. Journal of Mathematics and Physics, 53(5), 2012. https://doi.org/10.1063/1.4711830.

[35] V. Pata. Stability and exponential stability in linear viscoelasticity. Milan Journal of Mathematics, 77:333-360, 2009. https://doi.org/10.1007/s00032-0090098-3.

[36] A. Pazy. Semigroups of linear operators and applications to partial differential equations. Springer-Verlaag, 1983. https://doi.org/10.1007/978-1-4612-5561-1.

[37] J.E. Muñoz Revira and M. Grazia Naso. Asymptotic stability of semigroups associated with linear weak dissipative systems with memory. Journal of Mathematical Analysis and Applications, 326(1):691-707, 2007. https://doi.org/10.1016/j.jmaa.2006.03.022.

[38] J.E. Muñoz Rivera. Asymptotic behaviour in linear viscoelasticity. Quarterly of Applied Mathematics, 52:628-648, 1994. https://doi.org/10.1090/qam/1306041.

[39] B. Said-Houari, S.A. Messaoudi and A. Guesmia. General decay of solutions of a nonlinear system of viscoelastic wave equations. Nonlinear Differential Equations and Applications (NoDEA), 18:659-684, 2011. https://doi.org/10.1007/s00030011-0112-7.

[40] A. Youkana. Stability of an abstract system with infinite history. arXiv preprint arXiv, 2018. 